\title{
Beyond the urban mask
}

\section{Local climate zones as a generic descriptor of urban areas - Potential and recent developments}

\author{
Benjamin Bechtel, Olaf Conrad, \\ Matthias Tamminga \\ CEN, Universität Hamburg \\ Hamburg, Germany \\ benjamin.bechtel@uni-hamburg.de
}

\author{
Matthias Demuzere \\ LHWM, UGhent, \\ Ghent, Belgium
}

Yong $\mathrm{Xu}$, Chao Ren, IOFC, CUHK

Hong Kong, China

\author{
Verdonck, M.L., Van Coillie, F. \\ FORSIT, UGhent \\ Ghent, Belgium \\ Linda See \\ IIASA \\ Laxenburg, Austria \\ Gerald Mills \\ School of Geography, UCD \\ Dublin, Ireland \\ Arthur Cassone \\ CNRS-U. de Strasbourg, France
}

\author{
Devis Tuia \\ GIUZ, University of Zurich \\ Zurich, Switzerland
}
Patrícia Lopes, Cidália C. Fonte
INESC, University of Coimbra
Coimbra, Portugal

\author{
N. Kaloustian, \\ ALBA, Université de Balamand, \\ Beirut, Lebanon
}

\begin{abstract}
Despite the great importance of cities, relatively little consistent information about their internal configuration (structure, cover and materials) is available. The World Urban Database and Access Portal Tools (WUDAPT) initiative aims at the acquisition, storage and dissemination of data on the form and function of cities indifferent levels. At the lowest level, the Local Climate Zone (LCZ) scheme provides a basic description of urban structure. This scheme is a climate-based typology of urban and natural landscapes that also provides relevant information on basic physical properties of the landscape, which can be used in modelling and observational studies. The LCZ scheme has large potential as a standard generic description of urban areas. In this paper the scheme and our standard mapping approach are presented, followed by recent improvements and research on object-based image analysis, transferability of trained LCZ classifiers, quality of crowd contributions, and the use of other data sources and methods.
\end{abstract}

Keywords-Local Climate Zones, WUDAPT, LULC, urban

\section{INTRODUCTION}

Despite the great importance and heterogeneity of urban areas in climate change mitigation and adaptation, relatively little consistent information about their internal configuration (such as structure, cover and materials) is available. Currently, most global databases created from Earth Observation data provide an urban mask without any internal differentiation [1], [2]. A suitable approach for increasing the information content is the spatial decomposition into urban structural types (UST) [3], but existing studies lack standardization and consistency in the definition of these types. Hence a common and generic description of urban structures is urgently needed as a first step towards a universal mapping that characterizes the internal configuration of cities.
The World Urban Database and Access Portal Tools (WUDAPT) initiative is an international collaborative project for the acquisition, storage and dissemination of climate relevant data on form and function of cities by remote sensing and crowdsourcing. In contrast to existing global urban mapping projects, WUDAPT focuses on the internal make-up of cities [4]. Form refers to a large set of urban canopy parameters, in particular those that comprise urban cover (land cover, vegetation type, vegetation organization), urban geometry (building height, width of streets, contiguous or isolated buildings, roof geometry), and materials (wall type, roof type, window type, road materials, window fraction on the wall, colour/albedo). Urban function addresses the metabolism of cities or how the inhabitants use the infrastructure (e.g. traffic and building energy use).

WUDAPT employs a hierarchical approach to urban data gathering; each higher level is associated with greater detail but each employs a protocol to ensure maximum consistency and comparability. The WUDAPT level 0 data product applies the Local Climate Zone (LCZ) scheme [5] for a rough discretisation of urban areas (see next section). Level 1 data provide greater detail, where more specific information and additional aspects of form and function are collected via crowdsourcing techniques. Level 2 provides urban data gathered at a finer spatial scale across the entire urban area ('wall-to-wall' coverage), ready to use in (urban) climate and energy balance models, among others.

WUDAPT is designed as a community-based project and, as such, is still in its early stages. In the era of increasing coverage, accuracy and resolution of free Earth Observation data, urban remote sensing can substantially contribute to the acquisition, especially of level 0 and level 2 data. 
TABLE I. ABRIDGED DEFINITIONS FOR LOCAL CLIMATE ZONES. FROM [5]. CAMERICAN METEOROLOGICAL SOCIETY. USED WITH PERMISSION.

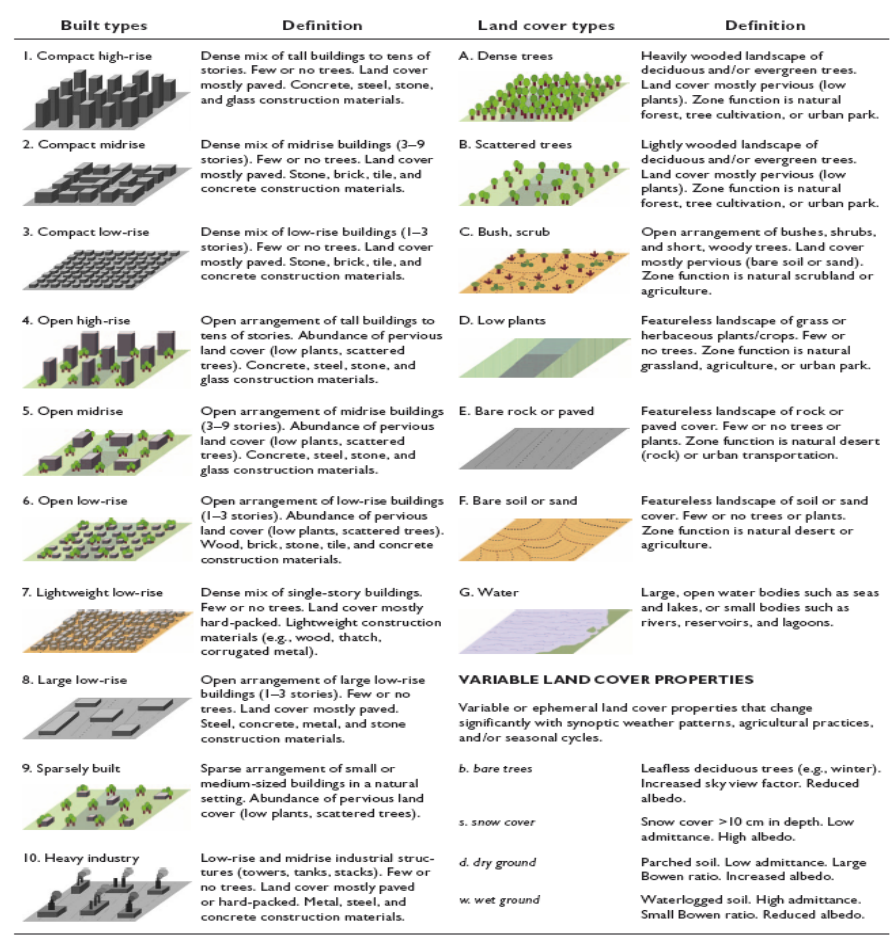

The aim of this paper is twofold. First, the LCZ scheme is introduced to stress its potential as a standard description of urban areas. Subsequently, the current mapping methodology is outlined. Second, shortcomings and recent improvements and developments are presented.

\section{LOCAL ClimAte ZONES MAPPING}

LCZs are a generic, climate-based typology of urban and natural landscapes. They were first introduced to standardize the classification of urban and rural field sites for observational urban heat island studies [5], and to overcome the urban rural dichotomy and the variety of landscapes assigned to either of them in case studies. LCZs are defined as "regions of uniform surface cover, structure, material, and human activity that span hundreds of meters to several kilometers in horizontal scale" [5] and have a "characteristic screen-height temperature regime". The basic scheme consisting of 10 urban and 7 natural classes is displayed in Table 1 .

We consider LCZ suitable as basis for a worldwide database of form and function of cities. In particular, LCZ are designed to be inclusive of all regional/cultural urban forms; eschews excessive detail in favour of basic physical descriptors of urban layout and; have been widely validated in urban climate studies worldwide. Furthermore, from an operational viewpoint, the LCZ types are well documented; each type is accompanied by a fact sheet with street view and bird's eye example images as well as numerous parameters related "to surface structure (sky view factor, aspect ratio, roughness element height), surface cover (plan fraction occupied by buildings, vegetation, and impervious ground), surface fabric (thermal admittance, surface albedo), and human activity (anthropogenic heat output)".

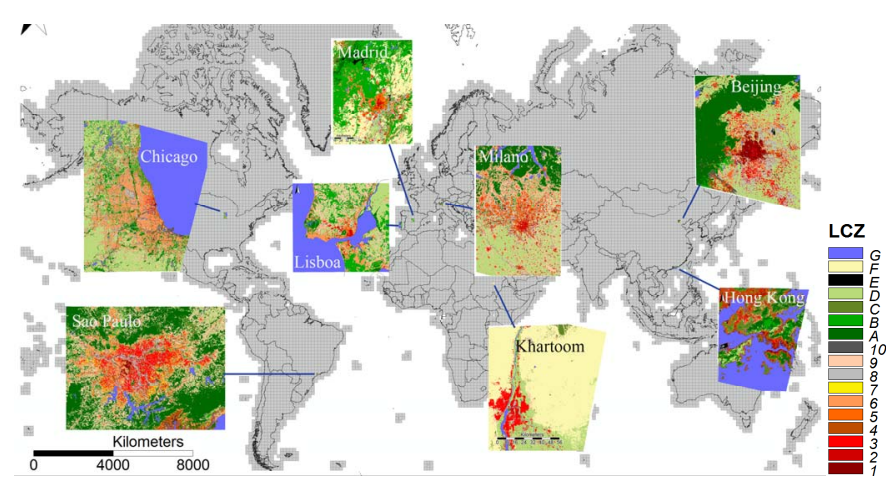

Fig. 1. Local Climate Zone classifications of selected cities (WUDAPT.org) Reprinted from [6]. (C) Creative Common Attribution 3.0.

In [7] the suitability of LCZs for mapping has been discussed and the scheme was found to quickly deliver substantial information on the basic physical properties of an urban area. Several mapping methods have been proposed including GIS-based approaches based on building models [8], object-based image analysis [9] and supervised pixel-based classification [10]. WUDAPT has reviewed various approaches under the following tenets: universality, free availability of data and software, objectivity, computational costs, and ease to apply [7]. A standard workflow has been developed using multi-temporal Landsat imagery, training data digitised in a Google Earth template and a Random Forest classifier. The algorithm is implemented in the open source software SAGA [11] and has been applied to about 50 cities (selected results shown in Fig. 1). The immensely different structures underline the need for differentiation beyond the built-up area.

Developing a globally applicable methodology LCZ remains a challenging task. In particular, the current method depends on high quality training data for each city due to the specific materials and bio-physical backgrounds. This paper examines how to improve the level 0 data by considering: the transferability of training area information between cities (section III.A); evaluating the quality and impact of the crowdsourced training data (III.B); cross comparison with other datasets to provide quality assurance (III.C) and; the potential for new datasets (III.D) or methods (III.E).

\section{RECENT INNOVATIONS IN LCZ MAPPING}

\section{A. Transferability of training areas}

The potential for transferring training labels from one city to another is practically limited by different bio-physical backgrounds, altitudes and acquisition dates. This was for example observed for the case of Beirut and Damascus in [12].

Some of these issues could be overcome using Google Earth Engine (GEE), which is a powerful cloud platform for online processing and visualisation of large geospatial datasets on Google servers and is free for research and education. Training and evaluation polygons can be imported or directly digitized via the GEE script editor, and general purpose classifiers, as well as various quality assessment procedures, are already available in GEE. The availability of image of Landsat and Sentinel (among other sensors) archives in GEE 
also might simplify the WUDAPT workflow and eases the comparison of different sensors for LCZ mapping and the creation of more generic features (e.g. cloud-free, homogeneously sampled, monthly median reflectance from all acquisitions). These can be used to train classifiers for one city and subsequently transfer it to others that are comparable in terms of morphology/materials and climate background. This transferability is currently being tested in Belgium and can reduce the need for city-specific training area samples and support the development of region-specific prototypes.

Developing classifiers that are robust enough to be transferred into different geographical, cultural and climatic conditions is also the aim of the 2017 Data Fusion Contest [13], organized by the Image Analysis and Data Fusion Technical Committee of the IEEE Geoscience and Remote Sensing Society. The challenge is organized in two rounds: first participants receive a set of data and training labels from a selection of cities while in the second, they receive only the data for other cities and are invited to upload classified maps.

\section{B. Quality of crowd contributions}

While the LCZ scheme provides an intuitive and culturally neutral description of urban neighbourhoods, experience has shown that creating suitable training areas for image processing is not straight-forward. The influence of the choice of training areas has a significant impact on LCZ map generation. This is because the concept of LCZs is more complicated than expected and expert knowledge is needed to distinguish classes from satellite imagery. Furthermore, human interpretation differs between operators, which is a general problem in remote sensing. To measure the error related to the choice of training areas and assess the quality of training areas without detailed ground reference, the HUman INfluence EXperiment (HUMINEX) was designed.

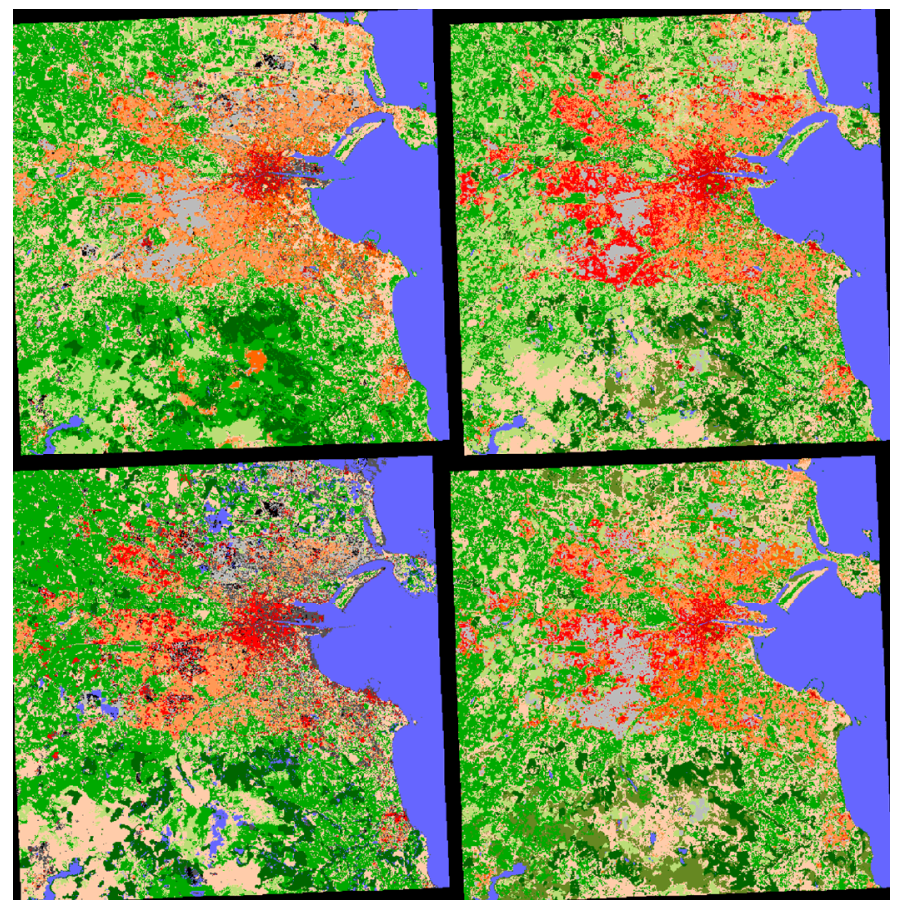

Fig. 2. Classifications from HUMINEX for Dublin, Ireland.
Specifically, student courses from 7 Universities generated test samples for different cities to quantify the impact of the operators' backgrounds on the classification accuracy. Preliminary results indicate that the human influence is considerable (see the example from Dublin in Fig. 2), thus raising the need for strict quality control.

\section{Cross-comparion}

In [6] the LCZ scheme was compared with Global Human Settlement Layer (GHSL) LABEL, an experimental product that extends the GHSL urban mask developed by the Joint Research Centre [2] to multiple-class land-cover. The built-up areas are thereby differentiated using different training data, and further reclassified using both the NDVI and the volume of buildings estimated from SRTM and ASTER-GDEM data. The comparison showed promising agreement at the city and $1 \mathrm{~km}$ scale (based on correlation coefficient, mean absolute distance, spatial pattern and radial distribution) but not at the pixel scale, mainly due to the mismatch in the grid scale and typology. In particular, open and compact classes agreed well.

\section{New datasets and features}

$\mathrm{Xu}$ and Ren investigated the use of both Landsat and ASTER data in LCZ mapping [14] for two high-density Chinese cities. They found that including textural information from both Landsat and ASTER data can achieve much better results than the conventional LCZ mapping method that uses spectral information from Landsat only. The overall prediction accuracies for Guangzhou and Wuhan showed accuracies of $66 \%$ and $84 \%$, respectively, compared to $62 \%$ and $81 \%$. When comparing the sensor data, Landsat data outperformed the ASTER data because of the additional spectral information (only VNIR from ASTER was used). The results were assessed for two major LCZ categories, including urban types (LCZ 110) and natural types (LCZ A-F). It was found that the overall prediction accuracy for urban types is still unsatisfactory when using optical data. This finding indicates that high-density urban areas are likely to have low LCZ mapping accuracy. To solve this, the use of interferometric SAR might be considered.

Kaloustian et al. also tested different input data for a case study in Beirut [12]. They found that all multi-spectral feature sets achieved good results, while feature sets with solely thermal or SAR data performed poorly. The latter is consistent with the results obtained for Khartoum in [15].

Additional tests are under development using data from OpenStreetMap (OSM) to assist the classification. The developed methodology requires the association of the features available in OSM and their spatial density with specific LCZ classes. Often OSM data does not provide enough information to discriminate between all urban classes (e.g. by building height), but it is possible to identify a subset (e.g. compact midrise or compact low-rise). Therefore LCZ maps for different seasons were derived and the OSM extracted data was used to eliminate classification inconsistencies for the city of Coimbra. The results indicated improvement of the classification, mainly of the natural land cover classes (A to F) [16]. 


\section{E. New apporaches for LCZ classification}

Casonne [17] compared the pixel-based approach with an object based image analysis (OBIA, implemented in SAGA) methodology using Beijing, Chicago, Dublin, Khartoum, and Madrid as case studies. A region growing image segmentation was applied on the Landsat- 8 data in $30 \mathrm{~m}$ resolution. Then multi-spectral features and attributes related to texture, band statistics, band ratios such as NDVI and form parameters were assigned. Segmentations with different granularity as well as different feature sets were tested. For validation, a different set of training areas was created. Overall, the object-based and pixel-based accuracies were comparable but the additional attributes slightly improved the classification (c.f. Fig. 3 for comparison of all feature sets). Furthermore, the fact that segmentation was performed at different scales can be exploited to generate LCZ maps at different scales.

Verdonck et al. assessed the influence of neighbourhood information on LCZ mapping using three cities in Belgium [18]. A contextual classifier was adopted to include neighbourhood information in the mapping process. The feature set was then extended by spectral statistics (mean, min, max, median, and quantiles) from the probability density distribution of each spectral band within a neighbourhood using a moving window. This resulted in improved discrimination, especially for the built-up classes. The overall accuracies increased by $10.3 \%, 6.1 \%$ and $5.0 \%$ compared to the standard methodology for Brussels, Antwerp and Ghent using kernels of 9x9, 9x9 and 7x7 $30 \mathrm{~m}$ pixels, respectively. The specific overall accuracies were of $92.9 \%, 93.7 \%$ and $95.3 \%$, which demonstrates the effectiveness of neighbourhood information in LCZ classification and is considered promising for further research of LCZ mapping in heterogeneous cities.

Montanges et al. [3] also studied the effect of accounting for spatial neighbourhood interaction. They explored the effectiveness of a Markovian model favouring nearby cells to take similar classes. Accounting for smoothness in the spatial domain greatly improved the classification accuracy of a model trained on satellite derived features, height descriptors, OSM roads and interest point densities. Tuia et al. [19] extended these findings by accounting for two interactions observed spatially: First some classes tend to attract each other while other repulse, second for scaling laws well known in geography, such as the power-law distribution of settlements. Encoding these two types of interaction jointly in a Markovian model, they estimated the LCZ distribution in North RhineWestphalia (Germany) and showed that such prior knowledge is highly beneficial for $\mathrm{LCZ}$ mapping.

\section{CONCLUSIONS AND OUTLOOK}

The next generation of global urban mapping products should focus on the internal form and function of cities and not only built-up areas. LCZs are a generic typology for UST discrimination and thus could become a standard in the future. A supervised pixel-based methodology is available, but further research is urgently needed. Thus we invite the urban remote sensing community to contribute to method development in this field. Recent attempts using OBIA, online processing platforms, textural or neighbourhood information and data from different sensors showed promising results for future improvements. This will strengthen the methodological development of WUDAPT and give insight into the type of satellite information needed for a high-quality LCZ classification. Furthermore, efforts should include the direct derivation of urban canopy parameters as cover fractions (built, vegetation, impervious), building height, or building volume.

\section{REFERENCES}

[1] T. Esch, M. Thiel, A. Schenk, A. Roth, A. Muller, and S. Dech, "Delineation of Urban Footprints From TerraSAR-X Data by Analyzing Speckle Characteristics and Intensity Information," IEEE Trans. Geosci. Remote Sens., vol. 48, no. 2, pp. 905-916, Feb. 2010.

[2] M. Pesaresi et al., "A Global Human Settlement Layer From Optical HR/VHR RS Data: Concept and First Results," IEEE J. Sel. Top. Appl. Earth Obs. Remote Sens., vol. 6, no. 5, pp. 2102-2131, Oct. 2013.

[3] A. P. Montanges, G. Moser, H. Taubenböck, M. Wurm, and D. Tuia, "Classification of urban structural types with multisource data and structured models," in JURSE, 2015, pp. 1-4.

[4] G. Mills, J. Ching, L. See, B. Bechtel, and M. Foley, "An Introduction to the WUDAPT project," presented at the 9th International Conference on Urban Climate, Toulouse, 2015.

[5] I. D. Stewart and T. R. Oke, "Local Climate Zones for Urban Temperature Studies," Bull. Am. Meteorol. Soc., vol. 93, no. 12, pp. 18791900, Dec. 2012.

[6] B. Bechtel et al., "Towards consistent mapping of urban structures - Global Human Settlement Layer and Local Climate Zones," ISPRS - Int. Arch. Photogramm. Remote Sens. Spat. Inf. Sci., vol. XLI-B8, pp. 1371-1378, Jun. 2016.

[7] B. Bechtel et al., "Mapping Local Climate Zones for a Worldwide Database of the Form and Function of Cities," ISPRS Int. J. Geo-Inf., vol. 4, no. 1, pp. 199-219, Feb. 2015.

[8] J. Geletič and M. Lehnert, "GIS-based delineation of local climate zones: The case of medium-sized Central European cities," Morav. Geogr. Rep., vol. 24, no. 3, pp. 2-12, 2016.

[9] Q. Weng, Global Urban Monitoring and Assessment through Earth Observation. CRC Press, 2014.

[10] B. Bechtel and C. Daneke, "Classification of Local Climate Zones Based on Multiple Earth Observation Data," IEEE J. Sel. Top. Appl. Earth Obs. Remote Sens., vol. 5, no. 4, pp. 1191-1202, Aug. 2012.

[11] O. Conrad et al., "System for Automated Geoscientific Analyses (SAGA) v. 2.1.4," Geosci Model Dev, vol. 8, no. 7, pp. 1991-2007, Jul. 2015.

[12] N. Kaloustian, M. Tamminga, and B. Bechtel, "Local Climate Zones and Annual Surface Thermal Response in a Mediterranean City," in JURSE, 2017.

[13] D. Tuia, G. Moser, B. Le Saux, B. Bechtel, and L. See, "2017 IEEE GRSS Data Fusion Contest: open data for global multimodal land use classification," IEEE Geosci Remote Sens Mag, 2017.

[14] Y. Xu, C. Ren, C. Meng, E. Ng, and T. Wu, "Classification of local climate zones using ASTER and Landsat data for hight-density Cities (in review)," IEEE J. Sel. Top. Appl. Earth Obs. Remote Sens.

[15] B. Bechtel, L. See, G. Mills, and M. Foley, "Classification of Local Climate Zones Using SAR and Multispectral Data in an Arid Environment," IEEE J. Sel. Top. Appl. Earth Obs. Remote Sens., vol. PP, no. 99, pp. 1-9, 2016.

[16] P. Lopes, C. Fonte, L. See, and B. Bechtel, "Using OpenStreetMap data to assist in the creation of LCZ maps," presented at the JURSE, 2017.

[17] A. Casonne, "Deriving Local Climate Zones from Remote Sensing data," Master thesis, Université de Strasbourg, 2016.

[18] M. L. Verdonck, A. Okujeni, S. van der Linden, M. Demuzere, R. De Wulf, and F. Vancoillie, "A contribution to WUDAPT: influence of neighbourhood information on Local-Climate Zone mapping in heterogeneous cities," Int. J. Appl. Earth Obs. Geo-Inf., submitted.

[19] D. Tuia, G. Moser, M. Wurm, and H. Taubenböck, "Land use modelling in North Rhine-Westphalia with interaction and scaling laws," JURSE, 2017. 